\title{
APERTURE AND FIELD CONSTRAINTS FOR THE VACUUM SYSTEM IN THE LHC INJECTION SEPTA
}

\author{
M. Gyr, B. Henrist, J.M. Jimenez, J-M. Lacroix, S. Sgobba, CERN, Geneva, Switzerland
}

\section{Abstract}

Each beam arriving from the SPS has to pass through five injection septum magnets before being kicked onto the LHC orbit. The injection layout implies that the vacuum chambers for the two circulating beams pass through the septum magnet yokes at a flange distance from the chamber of the beam to be injected. Specially designed vacuum chambers and interconnections provide the required straightness and alignment precision, thus optimising the aperture for both the circulating and injected beams, without affecting the quality of the magnetic dipole field seen by the injected beam. The circulating beams are shielded against the magnetic stray field by using $\mu$-metal chambers with a thickness of $0.9 \mathrm{~mm}$ to avoid saturation of the $\mu$-metal $(0.8 \mathrm{~T})$, coated with copper $(0.4 \mathrm{~mm})$ for impedance reasons and NEG for pumping and electron cloud purposes. A $2 \mathrm{~mm}$ gap between the iron yoke and the $\mu$-metal chamber allows an in-situ bake-out at $200^{\circ} \mathrm{C}$, based on a polyimide/stainless steel/polyimide sandwich structure with an overall thickness of $0.2 \mathrm{~mm}$. The constraints are described and the resulting vacuum system design, the apertures and the residual stray field are presented in this paper.

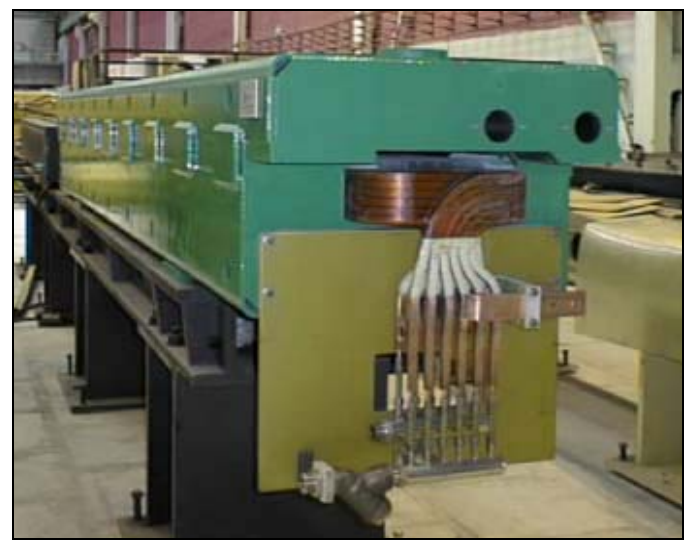

Figure 1: View of a $4 \mathrm{~m}$ long MSIB-module.

\section{INTRODUCTION}

Injection into LHC is performed in the combined experimental and injection insertions IR2 and IR8. The transfer lines TI 2 and TI 8 bring the beams to a point $\sim 150 \mathrm{~m}$ left of IP2 for injection into Ring 1 and $\sim 160 \mathrm{~m}$ right of IP8 for injection into Ring 2, respectively. In both insertions the beam approaches the LHC from outside and below the machine plane. The beams are directed by a last series of dipoles, already located in the LHC tunnel, towards a series of five Lambertson type septum magnets MSIA and MSIB (Fig. 1) which deflect the beam horizontally by $12 \mathrm{mrad}$ under the outer ring. A series of four kicker magnets then deflects the beams vertically by $0.85 \mathrm{mrad}$ onto the orbit. The LHC injection system [1] and the injection septum magnets MSI [2] are described in detail elsewhere.

\section{ALIGNMENT OF THE SEPTUM MAGNETS MSI}

To optimise the available aperture - especially for the injected beam - without making the vacuum design too magnet specific, the magnets will be tilted with respect to the machine plane and the MSIA and MSIB groups aligned such that the injected beams are centred vertically inside the $25 \mathrm{~mm}$ field gap.

Fig. 2 shows the vertical beam trajectory and envelope of the circulating and injected beams inside the two groups of septa in IR2. For the circulating beam a $9.8 \sigma_{y}$ beam envelope is shown, including $21 \% \beta$-beating, $27 \%$ parasitic dispersion, $\pm 4 \mathrm{~mm}$ orbit error and $\pm 2 \mathrm{~mm}$ mechanical and alignment tolerance (the mechanical tolerances of the chamber are treated as effective position errors of the beam). For the injected beam, a $5.5 \sigma_{\mathrm{y}}$ beam size is plotted, including $21 \% \beta$-beating and $\pm 1.5 \mathrm{~mm}$ mechanical and alignment tolerance.

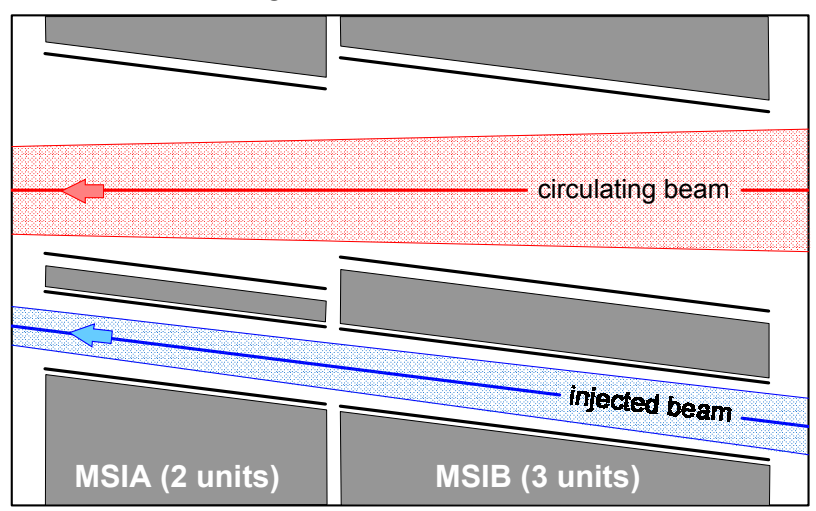

Figure 2: Vertical alignment of the MSI in IR2.

\section{APERTURE ISSUES IN THE MSI}

The effective apertures in the septa are determined by the vacuum chambers, the magnet alignment, the beam sizes and orbit offsets at these locations. The optimised vacuum chamber design described below and in [3] guarantees a beam stay-clear aperture of $52.4 \mathrm{~mm}$ for both circulating beams inside the vacuum tube with inner mechanical diameter $\emptyset_{\mathrm{i}}=57.2 \mathrm{~mm}$ (Fig. 5). This value takes into account all mechanical tolerances and errors, like straightness, cylindricity, alignment, etc.

The nominal trajectories of the circulating beams are not coaxial but vertically offset with respect to the axis of their vacuum chamber (Fig. 2). 

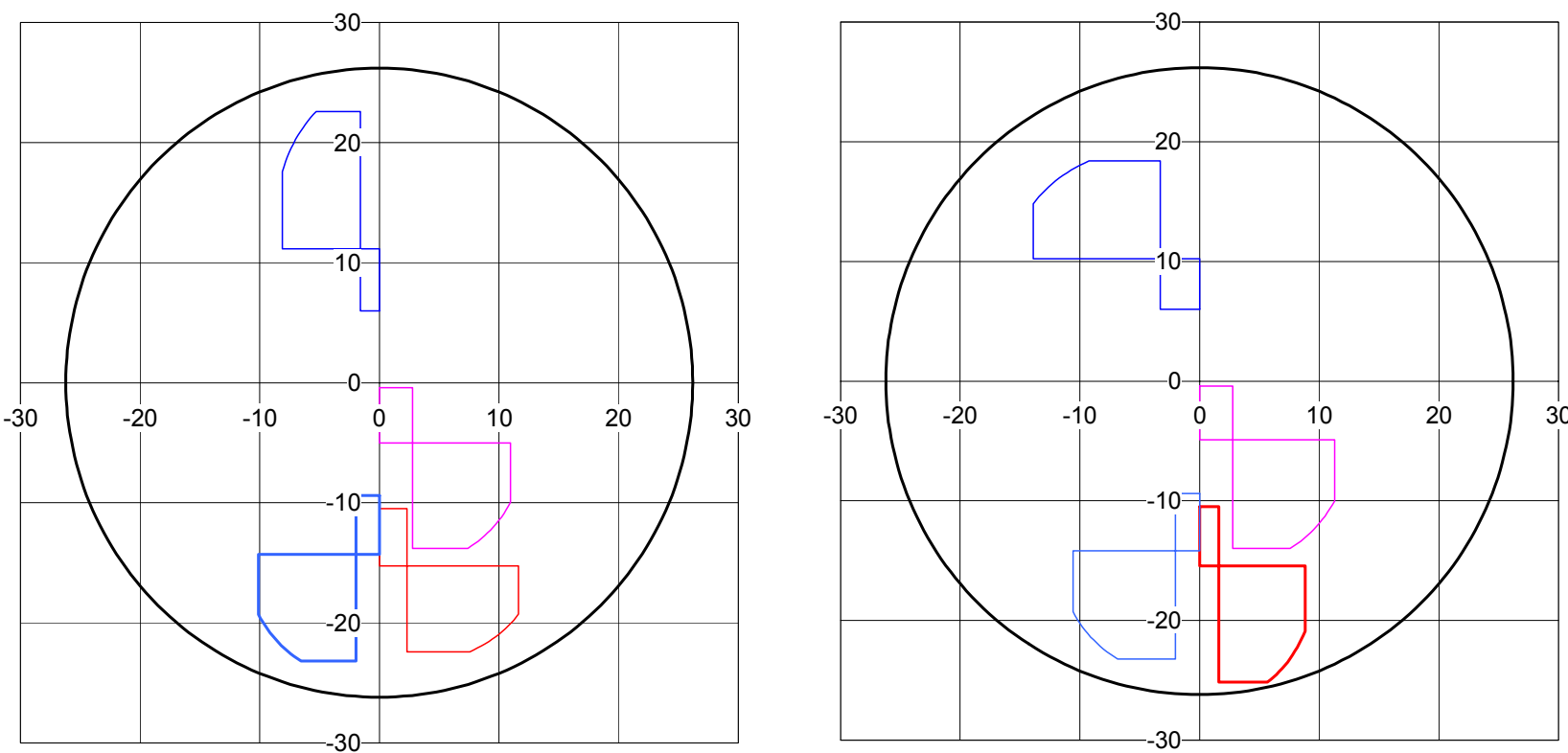

Figure 3: Aperture requirements for the circulating beams in the septum hole (left) and in the $2^{\text {nd }}$ beam hole (right) of the MSI in IR2. The geometrical shape of the secondary halo for the nominal normalised aperture $\mathrm{n}_{1}=7$ is plotted at the entrance and exit of the MSIB (left half-plane) and MSIA (right half-plane), respectively, and for the most unfavourable displacement $\Delta_{\mathrm{x}, \mathrm{y}}$. The circles represent the $52.4 \mathrm{~mm}$ stay-clear aperture of the chambers.

The geometrical shape of the secondary halo at the entrance and exit of the MSIB and MSIA, respectively, and for the nominal primary collimator aperture $\mathrm{n}_{1}=7$ as defined in [4] and [1] is plotted in Fig. 3.

The beam displacements $\Delta_{\mathrm{x}, \mathrm{y}}$ with respect to the nominal trajectory are defined by the momentum offset due to dispersion, the offset caused by the crossing separation and the closed orbit excursion [4]. They correspond to the latest optics and bump information. At each location the $\mathrm{x}, \mathrm{y}$-components of the $4 \mathrm{~mm}$ radial orbit and the signs are chosen such as to point towards the most unfavourable direction, i.e. closest to the aperture limit: $\Delta_{\mathrm{y}}>0$ for nominal beam positions above the axis of the vacuum chamber, $\Delta_{\mathrm{y}}<0$ otherwise. Since the nominal beam position is centred horizontally inside the vacuum chamber, $\Delta_{\mathrm{x}}$ has been chosen arbitrarily positive for the beams in the MSIA and negative for the beams in the MSIB.

It can be seen that the beam in the septum hole and in the $2^{\text {nd }}$ beam hole both pass, albeit less comfortably in the latter one where the margin for $\mathrm{n}_{1}$ is less than $4 \%$. To overcome this aperture bottleneck, the original V6.5 optics needed to be slightly modified. The situation is somewhat more comfortable for the injection in IR8, where there is enough aperture for $n_{1}>8$.

\section{VACUUM SYSTEM}

In the injection septa, the circulating beams are passing through the magnet yoke where they would be exposed, without shielding, to a leaking magnetic stray field of up to 30 Gauss (Fig. 4). This is unacceptable and has to be suppressed by about a factor of 10 . The finally adopted chamber concept and modular pumping ports resulted from the superposition of several requirements: the shielding against magnetic stray fields, the aperture, the impedance, the radiation, the alignment tolerances, the tilt of the magnets and the different vacuum performances.

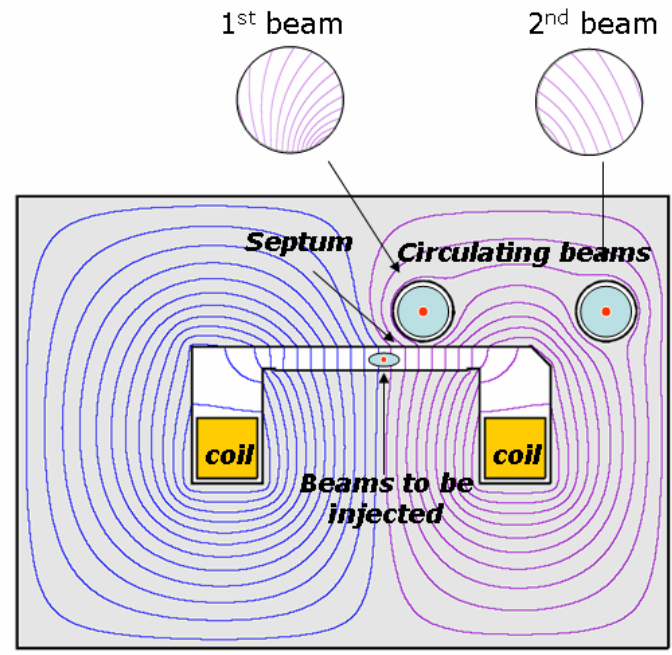

Figure 4: Schematic view of the cross-section of an MSIA septum magnet. The residual stray field in the holes for the circulating beams is to be shielded with a $\mu$-metal vacuum chamber.

The outer diameter being fixed by the diameter of the hole in the magnet yoke $\left(\varnothing=64^{ \pm 0.1} \mathrm{~mm}\right)$, less than about $4 \mathrm{~mm}$ on the radius were available for the vacuum chamber wall, copper coating, bake out heaters, mechanical tolerances and gap of insertion to match the required aperture. A $\mu$-metal (Ni $76.43 \%$, Fe $13.83 \%$, Cu $5.04 \%$, Mo $4.02 \%$ Alloy) chamber has been chosen since it pro- 
vides shielding against leaking stray fields while saving aperture for the circulating beams.

This material, known for its shielding properties after a heat treatment at $1050^{\circ} \mathrm{C}$, has an intrinsic limitation, as its magnetic permeability is dramatically reduced $(-70 \%$ measured on the welds) by any weld or plastic deformation inducing constraints in the manufacturing process. However, reducing the number of welds is anyway interesting because it prevents leaks due to radiation stimulated corrosion (the injection areas are potentially radioactive areas).

The procurement of the $\mu$-metal chambers has been completed with the following geometrical tolerances: $0.2 \mathrm{~mm}$ out of roundness, $0.2 \mathrm{~mm}$ tolerance on average diameter, $\pm 0.075 \mathrm{~mm}$ tolerance on the thickness and $1 \mathrm{~mm}$ straightness over 5 meters. The $\mu$-metal thickness resulted from measurements in a real magnet which showed that the saturation of the $\mu$-metal $(0.8 \mathrm{~T})$ with a $2 \mathrm{~mm}$ gap between the pipe and the magnet bore is avoided with a minimum thickness of $0.9 \mathrm{~mm}$ [5].

The impedance contribution has been reduced by an inner copper coating $(0.4 \mathrm{~mm}$ electrodeposited, Fig. 5) providing an electrical resistance of $1.9 \times 10^{-4} \Omega / \mathrm{m}$; scratch tests confirmed the absence of delamination between the copper layer and the $\mu$-metal [6].

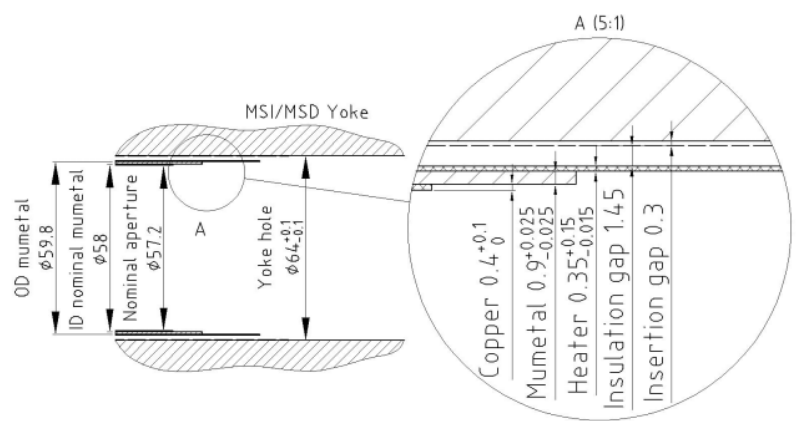

Figure 5: Schematic view of the $\mu$-metal pipe configuration in the magnet yoke.

In the welding area, when using 316LN flanges (LHC standard), nitrogen is released from the austenite matrix and gas bubbles are formed imposing the use of 304L (or $316 \mathrm{~L}$ ) flanges. In fact, the poor "weldability" between $\mu$ metal and nitrogen added austenitic stainless steel (316LN) results from the low concentration of $\mathrm{Cr}$ and $\mathrm{Mn}$ which help in the stabilisation of nitrogen [7-9].

As part of the LHC room temperature vacuum system, the $\mu$-metal chambers shall also fulfil the Ultra High Vacuum requirements (in terms of leak tightness and low outgassing) to achieve the required 100 hours beam lifetime $\left(<10^{-7} \mathrm{~Pa}\right.$ dynamic pressure), avoid vacuum instabilities and limit the background to the experiments downstream the injection areas (Alice and $\mathrm{LHCb}$ ) resulting from the beam gas scattering.

To achieve such UHV vacuum, both the cleanliness of the inner surfaces at all stages of assembly work and a bake-out are required. The use of NEG (TiZrV) coatings provides an additional distributed pumping and serves as an electron cloud suppressor due to its low secondary electron yield after activation [10]. The magnetron sputtering of NEG coatings on an electrodeposited copper inside a $\mu$-metal chamber required a special procedure since the oxidisation of the copper surface was identified as a major problem for the NEG deposition [10].

For the bake-out, a $0.2 \mathrm{~mm}$ thick new heater concept based on a sandwich polyimide/stainless steel/polyimide [11] has been developed to achieve the minimum temperature of $200^{\circ} \mathrm{C}$ required to activate the NEG coatings while preventing the overheating of the magnet laminations $\left(100^{\circ} \mathrm{C}\right)$, the heat transfer being limited by the remaining $2 \mathrm{~mm}$ air gap and the use of a reflective film. Magnetic measurements confirmed that 10 bake-out cycles at $300^{\circ} \mathrm{C}$ during 24 hours does not decrease the magnetic permeability. This permanent bake-out will also be remotely controlled to reduce the radiation exposure.

\section{SUMMARY}

A technically quite demanding vacuum system has been developed to comply with all the stringent requirements (ultra high vacuum, magnetic shielding, low beam impedance, sufficiently large apertures, etc.) in the presence of a number of hard constraints (given beam hole diameter and gap height, mechanical and alignment tolerances, need for bake-out, etc.).

\section{REFERENCES}

[1] O. Brüning et al. (Eds)., "LHC Design Report, Vol. I", CERN-2004-003.

[2] S. Bidon et al., "Steel Septum Magnets for the LHC Beam Injection and Extraction", EPAC'02, La Vilette, June 2002, pp. 2514-2516.

[3] J.M. Jimenez, B. Henrist, "Vacuum Chambers for the Circulating Beams in the LHC Injection and Extraction Septa", Functional Spec LHC-VCRS-ES0001 rev. 1.0.

[4] J.B. Jeanneret, R. Ostojic, "Geometrical Acceptance in LHC Version 5.0", LHC Project Note 111, September 1997.

[5] M. Gyr, priv. comm. CERN-AB/BT and BINP, Protvino.

[6] A. Gerardin, "Scratch Test on an Electro-deposed Copper Layer of a $\mu$-metal Pipe", EST/SM-ME EDMS 349561 16/07/02.

[7] Report $\mathrm{N}^{\circ}$ 2001/07/010 EST/SM/JH/ph, Metallurgy section, CERN.

[8] Report $\mathrm{N}^{\circ}$ 2001/08/008 EST/SM/JH/ph, Metallurgy section, CERN.

[9] Y. Forestier, "Caractérisation d'une soudure 304L/ $\mu$ metal », CERN EDMS 331721, 10/12/2001.

[10] G. Andreolety et al., "The Septa Magnets Vacuum Chambers of the LHC: Production Procedure and Vacuum Characterization of the First Prototypes", EVC-9, Paris, April 2005.

[11] Ch. Rathjen et al., "Ultrathin Polyimide-Stainless Steel Heater for Vacuum System Bake-Out" these Proceedings, RPPE043. 